\title{
Correction to: Critical role of interleukin-23 in development of asthma promoted by cigarette smoke
}

\author{
Hyun Seung Lee ${ }^{1} \cdot$ Da-Eun Park ${ }^{1} \cdot \mathrm{Ji}^{-W}$ Won Lee ${ }^{2} \cdot \mathrm{Hoe}^{-N a} \mathrm{Kim}^{1} \cdot$ Woo-Jung Song ${ }^{2,3} \cdot$ Heung-Woo Park $^{4}$. \\ Sang-Heon $\mathrm{Cho}^{1,4}$
}

Published online: 22 May 2019

(C) Springer-Verlag GmbH Germany, part of Springer Nature 2019

\section{Correction to: Journal of Molecular Medicine https://doi.org/10.1007/s00109-019-01768-y}

The original publication of this paper contains a mistake.

Correct images for figures 1,2, 3, 4 and 5 are shown in this paper.

The original article has been corrected.

The online version of the original article can be found at https://doi.org/ 10.1007/s00109-019-01768-y

Hyun Seung Lee

gentlehood79@hanmail.net

1 Institute of Allergy and Clinical Immunology, Medical Research

Center, Seoul National University, 101 Daehak-ro, Jongno-gu,

Seoul 110-744, South Korea

2 Division of Allergy and Clinical Immunology, Department of Asan Medical Center, College of Medicine, University of Ulsan,

Seoul, Republic of Korea

3 Division of Allergy and Clinical Immunology, Department of Internal Medicine Asan Medical Center, College of Medicine, University of Ulsan, Seoul, Republic of Korea

4 Department of Internal Medicine, College of Medicine, Seoul National University, Seoul, Republic of Korea 


\begin{tabular}{|c|c|c|}
\hline \multicolumn{3}{|c|}{ Intranasal $\mathrm{Dp}$ and/or CSE } \\
\hline $\begin{array}{l}\downarrow \\
\text { D0 }\end{array}$ & $\begin{array}{l}\downarrow \\
\mathrm{D} 1\end{array}$ & \begin{tabular}{cc}
\multicolumn{2}{c}{ Sacrifice } \\
$\downarrow$ & $\downarrow$ \\
D2 & D3
\end{tabular} \\
\hline
\end{tabular}

b

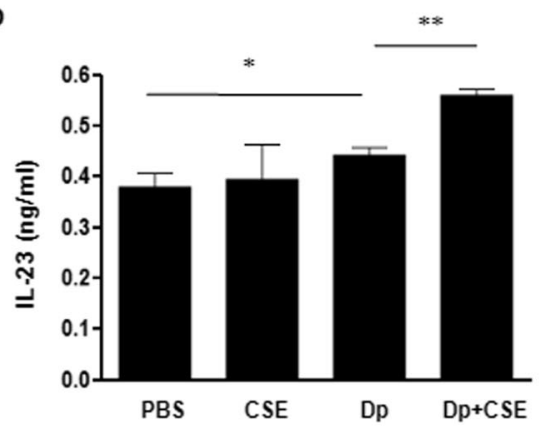

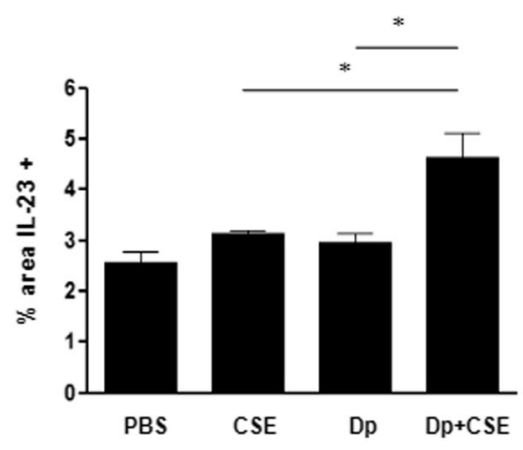

Isotype
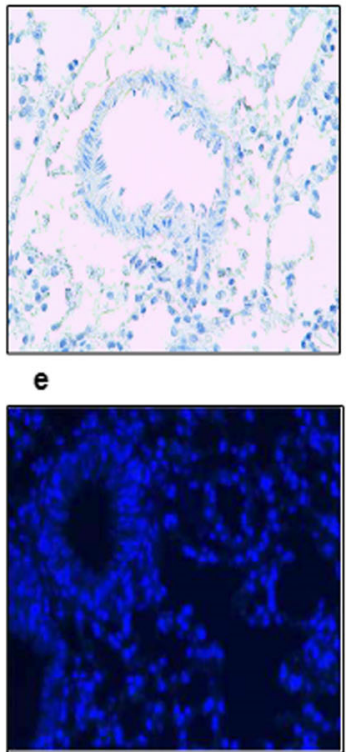

Green mean : 9.24

PBS

CSE

Dp

$\mathrm{Dp} / \mathrm{CSE}$
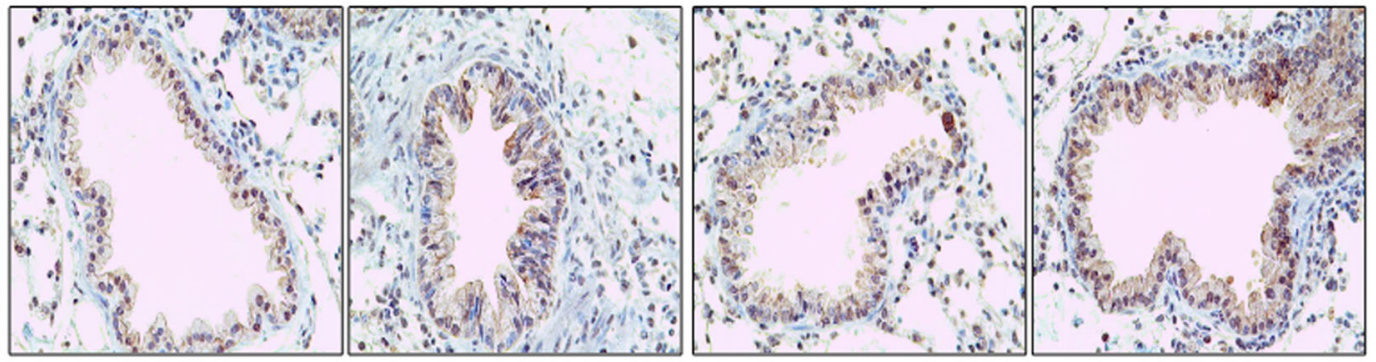

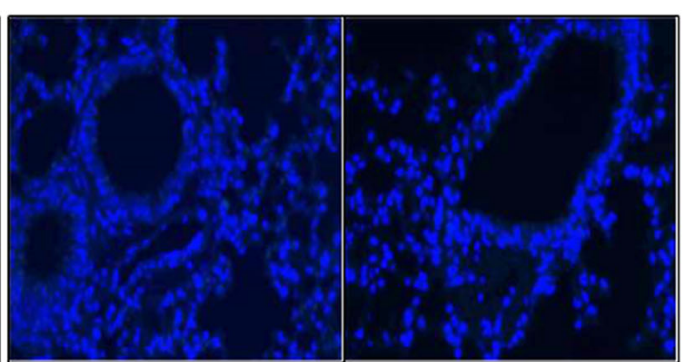

Green mean : 14.26

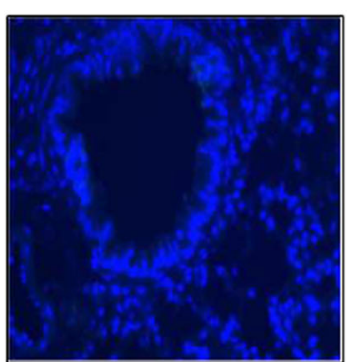

Green mean : 15.09

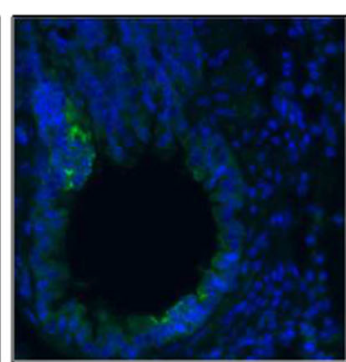

Green mean : 25.94

Fig. 1 The expression of IL-23 in lung of mice simultaneously exposed to PBS or Dp and 3 days of cigarette smoke extract (CSE). a Experimental protocol. The expression of IL-23 from lung was detected after instillation of $\mathrm{Dp}$ with or without CSE. $\mathbf{b}$ The protein level of IL-23

in crushed lung was detected using ELISA. $\mathbf{c}$ The expression of IL-23 in lung was detected using immunohistochemistry staining (IHC). d IL-23 IHC quantification. e The expression of IL-23 in lung was detected using immunofluorescence staining (IF, green). ${ }^{*} p<0.05, * * p<0.01$ 
a

\section{Sensitization, i.n.}

Challenge, i.n.

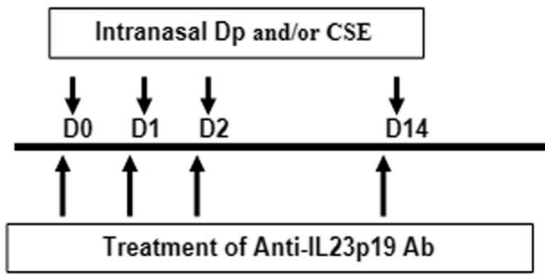

Dp Dp Dp

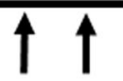

MBPT Sacrifice
D21 D22D23 D24 D25

b

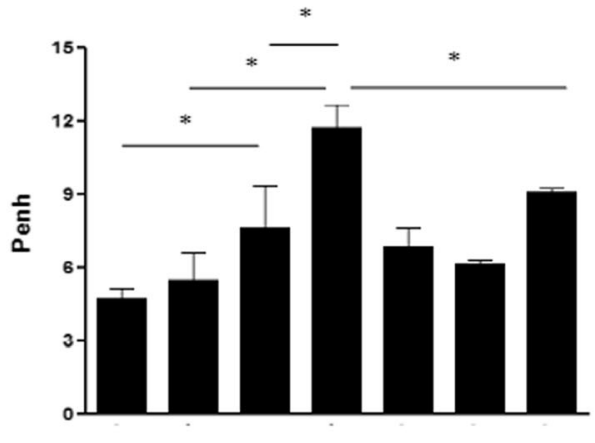

c

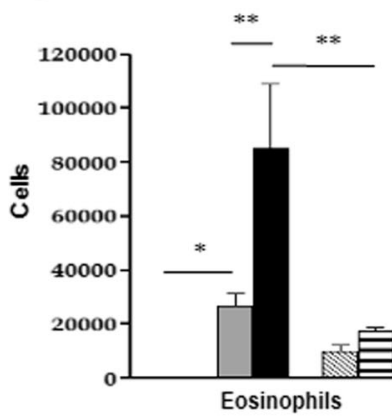

$\square$ PBS
$\square$ CSE

$\square$ Dp

$D p+C S E$

d

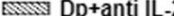

D Dp/CSE+anti IL-23

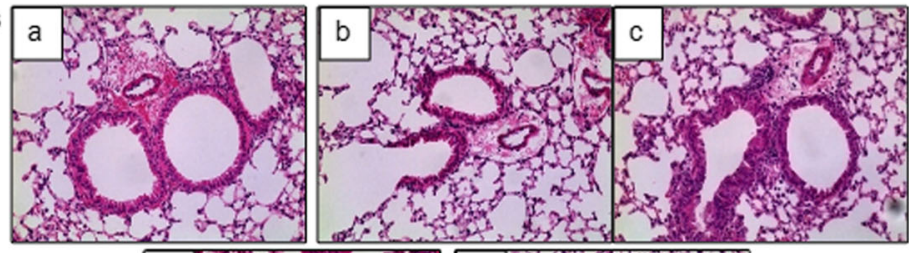

e

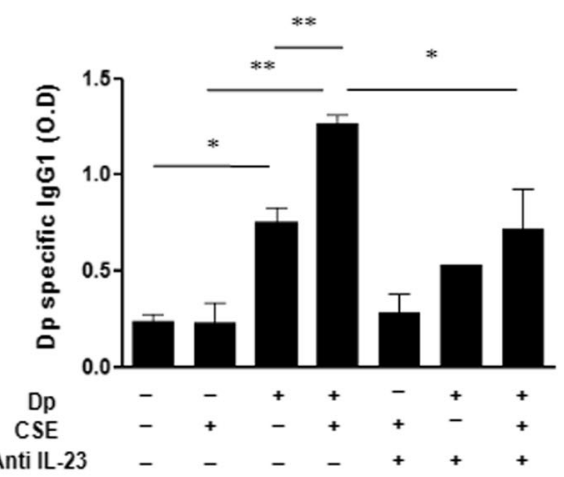

Fig. 2 The evaluation of asthmatic phenotypes in anti-IL-23 Ab-treated mice during sensitization period. a Experimental protocol for house dust mite and cigarette smoke extract induced asthma model of study. b Methacholine hyperresponsiveness was measured $24 \mathrm{~h}$ after the last challenge. $\mathbf{c}$ The numbers of eosinophils and neutrophils in BALF. d Lung
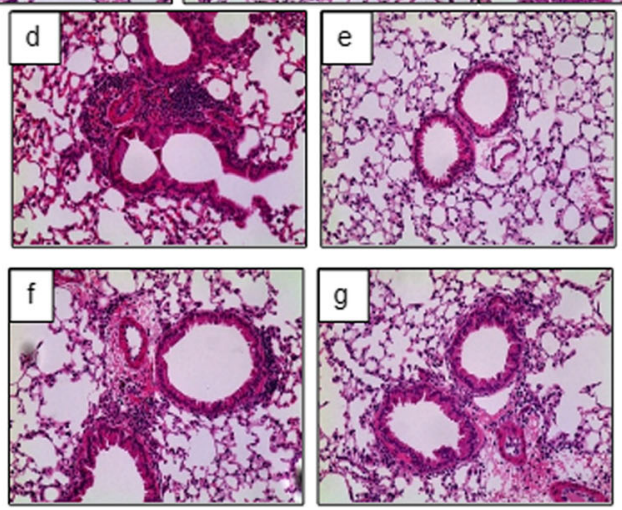

histology after the last challenge (a: PBS, b: CSE, c: Dp, d: Dp/CSE, e: CSE + anti-IL-23 Ab, f: Dp + anti-IL-23 Ab, g: Dp/CSE + anti-IL-23 Ab, H\&E stain, $\times 200)$. e Serum Dp-specific IgG1 was evaluated after the last challenge. OD, optical density; $* p<0.05 ; * * p<0.01$ 


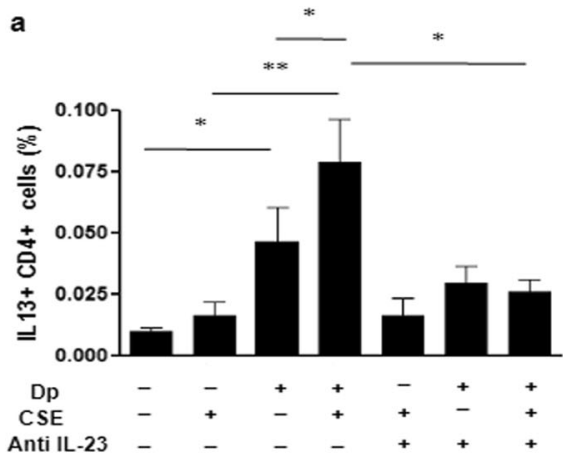

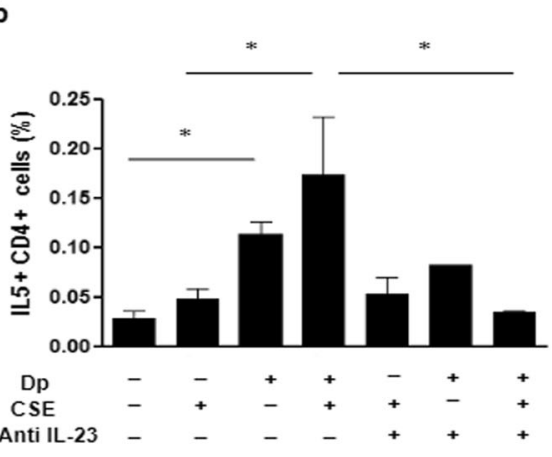

c

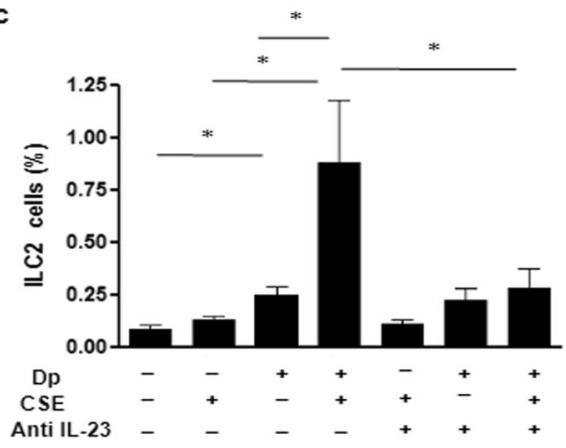

d

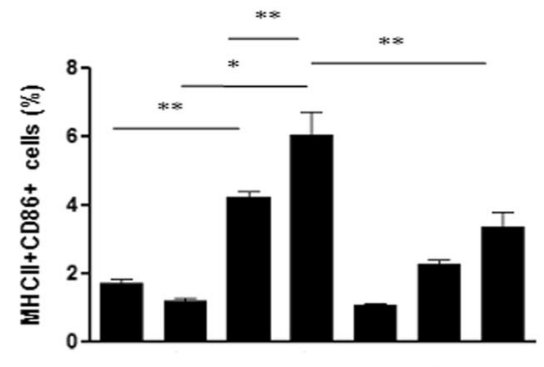

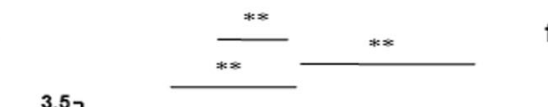

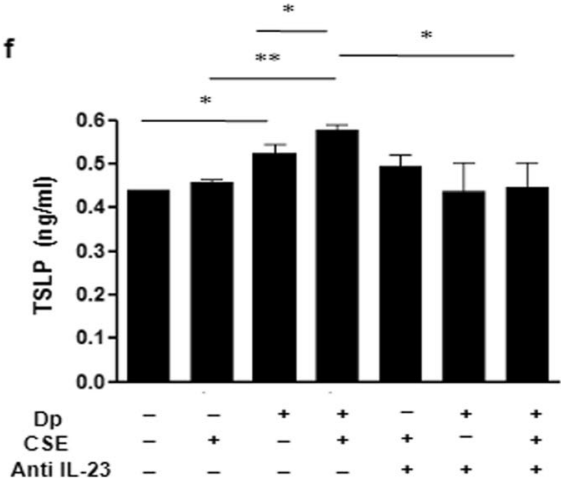

CSE

Anti IL-23

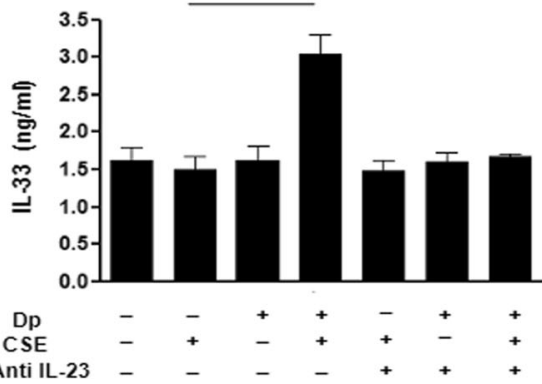

Anti IL-23

sacrificed $24 \mathrm{~h}$ after the last sensitization. After sacrifice, lung draining lymph nodes were isolated from mice. $\mathbf{d}$ The numbers of MHCII+ CD86+ cells in CD11c + DCs were determined using flow cytometry. e-f The levels of IL-33 and TSLP in the supernatants of crushed lungs were determined using ELISA; $* p<0.05 ; * * p<0.01$ 

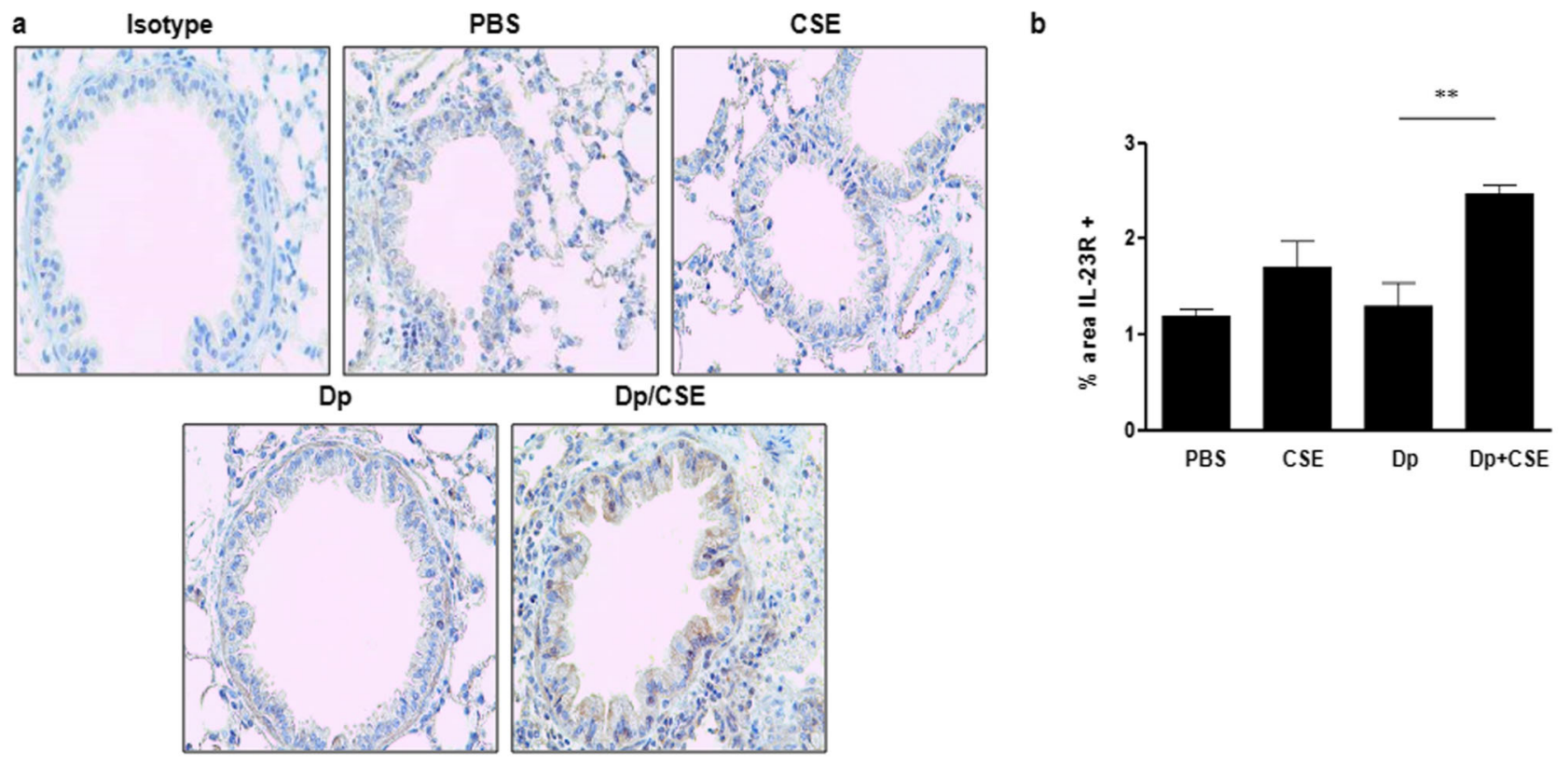

C

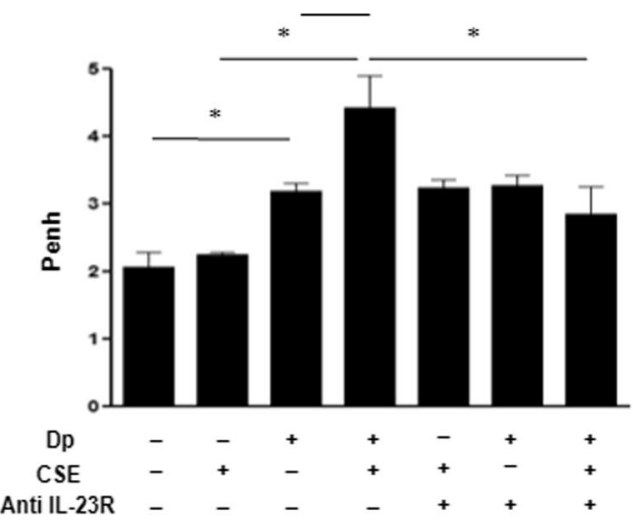

Fig. 4 The expression of IL-23R in lung of mice after sensitization and asthmatic phenotypes after challenge in anti-IL-23R Ab-treated mice during sensitization period. The expression of IL-23R in lung of mice simultaneously exposed to PBS or house dust mite and 3 days of cigarette smoke extract. a The expression of IL-23R in lung was detected using

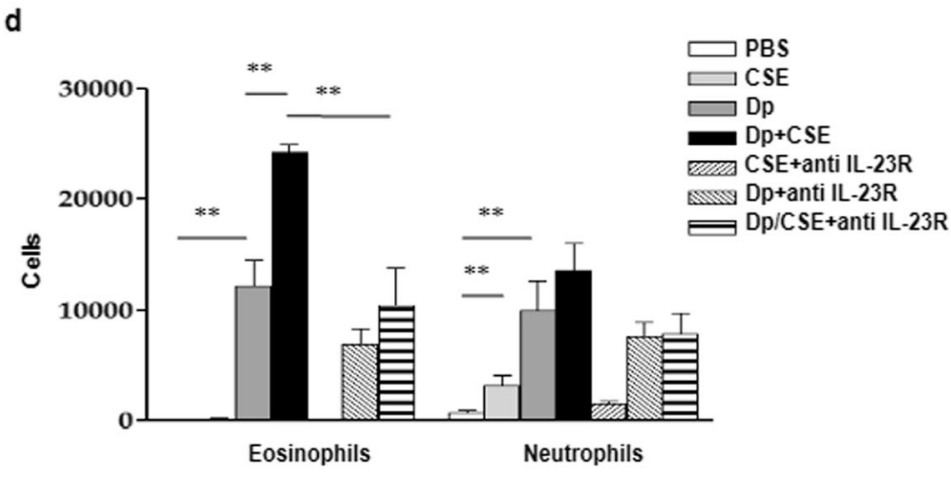

immunohistochemistry staining (IHC). b IL-23R IHC quantification. After treatment of anti-IL-23R Ab in the sensitization period, mice were sacrificed $24 \mathrm{~h}$ after the challenge. c Methacholine hyperresponsiveness was measured $24 \mathrm{~h}$ after the last challenge. $\mathbf{d}$ The number of eosinophils and neutrophils in BALF. $* p<0.05, * * p<0.01$ 

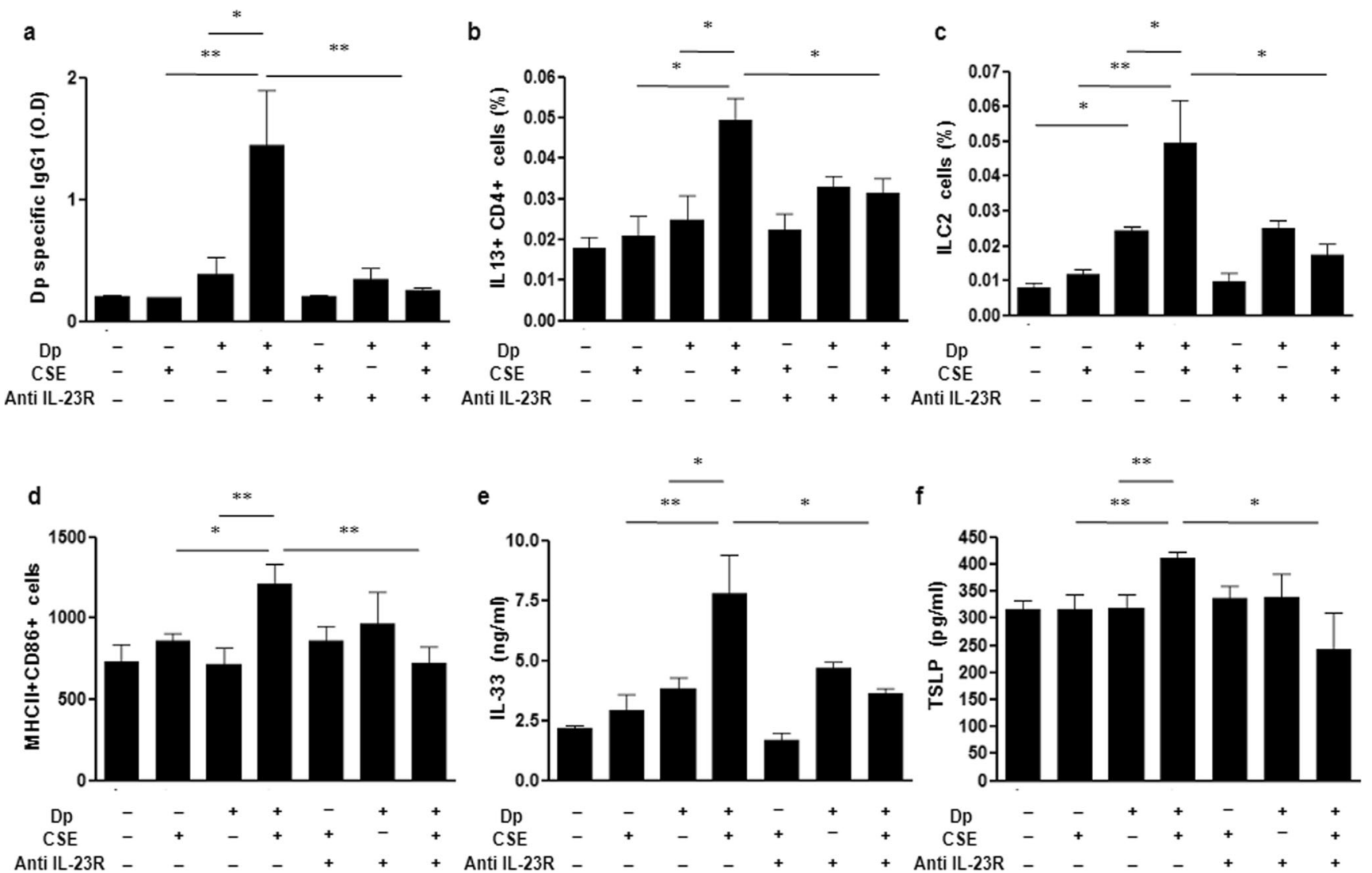

Fig. 5 The population of IL13+ CD4+ Tcells, IL13 + ILC2 after challenge and the numbers of DCs, the level of innate pro-Th2 cytokines after the last sensitization. a Serum Dp-specific IgG1 was evaluated after the last challenge in anti-IL-23R Ab-treated mice during sensitization. $\mathbf{b}$ c IL13- produced CD4+ T cells and IL13-produced type 2 innate lymphoid cell from lung were evaluated after the last challenge in anti-IL-23R Abtreated mice during sensitization. After treatment of anti-IL-23R Ab in

the sensitization period, mice were sacrificed $24 \mathrm{~h}$ after the last sensitization. After sacrifice, lung draining lymph nodes were isolated from mice. d The numbers of MHCII+ CD86+ cells in CD11c + DCs were determined using flow cytometry. e-f The levels of IL-33 and TSLP in the supernatants of crushed lungs were determined using ELISA. * $p<0.05$, $* * p<0.01$

Publisher's note Springer Nature remains neutral with regard to jurisdictional claims in published maps and institutional affiliations. 